\title{
Sistema de consejería en línea para la Universidad Técnica de Ambato: una aproximación al tema.
}

Online counseling system for the technical university of Ambato: an approach to the topic.

Teresa de Jesús Paredes Ruíz. ${ }^{1}$, Lorena del Carmen Chiliquinga Vejar. ${ }^{2}$, Malena Karina Quiroga López. ${ }^{3}$ \& Anita Dalila Espín Mininguano. ${ }^{4}$

\section{DOI: https://doi.org/10.33262/visionariodigital.v2i3.91}

\section{Resumen.}

La tecnología ha llegado a tener un papel protagónico en la llamada sociedad de la información por lo que no puede estar desligado de ningún área del quehacer humano. Los sectores sociales y culturales no son la excepción dado que su adecuada incorporación puede potenciar procesos y además fortalecer la calidad de vida de las personas.

"Dada la época actual en que el ser humano tecnológicamente vive, podemos observar que todas las personas están ligadas más naturalmente al computador, de ahí que "Cuanto más la tecnología avanza, más común, es observar la utilización de nuevas tecnologías que los profesionales de la área social se apoyan. El Internet es uno de los instrumentos tecnológicos usados en el asesoramiento psicológico. Con el avance de la utilización de aplicaciones de Internet, la asesoría en línea ha sido un fenómeno común a todos los consejeros." Tanrikulu (2009, pág. 2)

El servicio de consejería en línea actualmente existe en varios países. Este servicio aporta buenos resultados debido a que el usuario puede acceder directamente desde cualquier lugar donde exista un computador con acceso a internet, teniendo la opción de acceder en

\footnotetext{
${ }^{1}$ Universidad Técnica de Ambato, Facultad de Jurisprudencia y Ciencias Sociales, Ambato, Ecuador, tdj.paredes@uta.edu.ec

${ }^{2}$ Universidad Técnica de Ambato, Facultad de Jurisprudencia y Ciencias Sociales, Ambato, Ecuador, ldc.chiliquinga@uta.edu.ec

${ }^{3}$ Universidad Técnica de Ambato, Facultad de Jurisprudencia y Ciencias Sociales, Ambato, Ecuador, malenakquiroga@uta.edu.ec

${ }^{4}$ Universidad Técnica de Ambato, Facultad de Jurisprudencia y Ciencias Sociales, Ambato, Ecuador, ad.espin@uta.edu.ec
} 
un horario más amplio al que podría en forma presencial. Este servicio ya se ha implementado en otros países.

Este tipo de consejería es efectivo, por cuanto el usuario puede expresar sus sentimientos en forma más abierta, conservando su anonimato. La publicación de la Asociación Nacional de Trabajadores Sociales de EE.UU (2007, pág. 2) dedicó su número para analizar el funcionamiento de la consejería en línea que consiste principalmente en sesiones de chat, conversaciones en tiempo real de información a través de una computadora, correos electrónicos estructurados, grupos de discusión en línea, pre-sesión con cuestionarios siempre y cuando esté relacionado con las necesidades del usuario. Advierte que es necesario para su correcto funcionamiento requerir información demográfica de los clientes, proveer otros servicios de ayuda, políticas claras y sobre los tratamientos y sus limitaciones, políticas de privacidad, etc.

Coleman (2015, pág. 1) contextualiza como las trabajadoras sociales clínicas ven cambios muy rápidos en las forma en que ofrecen los servicios, ya que ahora se puede ofrecer los servicios vía internet, lo que se llama e-terapia, ciberterapia o consejería del internet. Se han realizado varias investigaciones sobre esta forma de ofrecer consejería, la cual ha dado resultados positivos motivando a trabajadoras sociales de otros países a usar este servicio.

La implantación de un sistema de consejería en la UTA, ayudaría a incrementar la cobertura de los servicios que actualmente ofrece el DIBESAU a los quince mil estudiantes que aproximadamente pertenecen a la institución, de esta forma se conseguiría mejorar la atención y seguimiento a los problemas emocionales que presenten.

Una aproximación inicial al tema, se refleja en la necesidad de contar con un servicio de consejería en línea que se tenga a acceso por medios telemáticos como chat, videoconferencia, entre otros. Sin embargo, a pesar de las ventajas ofrecidas por este tipo de sistema de consejería, de las investigaciones preliminares realizadas por el equipo de investigadoras, se concluye que no existe una metodología que oriente sobre la creación de este sistema y que ofrezca pautas claras sobre los procedimientos a aplicar por parte de los profesionales que la realizan o sobre el tipo de recursos tecnológicos a aplicar.

La Consejería en Línea tiene estrategias a utilizar tanto a nivel psicológico como tecnológico. Siendo una metodología que se puede poner a disposición de la Universidad Ecuatoriana y otras instituciones públicas o privadas que deseen implementar este sistema.

Palabras clave: Sistema, Consejería, Línea, Jurisprudencia, Ciencias Sociales

\section{Abstract.}


The technology has become very important in our digital society, being part of the everyday life of the human beings. The social actors and their culture are not exception to this; given it's right accommodation will result in a benefit process and build strengths to people's quality of lives.

In the present times, where the human being lives with technology, it can be observed their natural symbiotic relation with a computer. From this in more the technology advances, the more use is given in the social area. The internet is an instrument technologic that is used in the social and mental health assessment. With this advance the use of the internet in the counseling on line has been a phenomenon advance to the counselors. Tanrikulu (2009, pág. 2)

There are on-line counseling services in various countries. This service gives good results due to the fact that the clients can access directly from any part where there is a computer with internet access. The schedule times offers more advantage than the one to one in person counseling. This type of counseling is effective not only because the client can express their feelings more openly and in an anonymous way.

The National Association of Social Workers of the EE: UU. (2007,pag.2) analyzed the service on counseling on line, that consist in chat sessions, conversations on real time, obtaining informaction, structured e-mails. Discussion groups on line, pre-session using questionnaires that are related to the group o individual needs. The organization advice the need to collect client's information, provide other help services, have clear policies on the treatment, and be clear about the limitation of the services, privacy.

Coleman (2015, pág. 1) observed how the clinical social workers see changes in the way the offer their services thru e-therapy, cyber therapy, on line counseling. The research on this type of counseling gives positive outcomes, motivating other social workers in other countries to do the same.

The implementation of the on-line counseling in the Universidad Técnica de Ambato will help to increment of the service coverage that actually offers de Students Services Department, with the goal to offer better services to the students that have emotional problems.

Still the benefits of the on line counseling, there is no evidence of methodologies that could direct on how to create this system and there are not clear procedures on how the professionals could use the technologic resources .

On line counseling can provide social and mental assessments together with new technological strategies created with the objective to serve the Ecuadorian university, public and private institutions that wish to implement this system. 
Keywords: System, Counseling, Line, Jurisprudence, Social Sciences

\section{Introducción.}

La sociedad de la información exige cada vez más profesionales con ciertas destrezas, conocimientos y actitudes. El área del Trabajo Social no ha quedado al margen de estas exigencias y cada vez es más notoria la necesidad de una sólida formación en el campo de la tecnología por parte de trabajadores sociales. "Uno de los retos a los que se enfrenta el Trabajo social en los próximos años se vincula con la capacidad de adaptación de la intervención social a la sociedad de la información" (Raya Diez \& Santolaya, 2009)

La consejería profesional en línea es una relación entre el profesional y los usuarios que se lleva a cabo por medios tecnológicos, incluye un proceso de ayuda, que garantiza que se mantenga las normas éticas establecidas en el campo profesional referentes a la seguridad y confidencialidad. Este tipo de consejería ofrece muchas ventajas al usuario, entre ellas se destaca la ayuda a personas que tienen miedo al estigma social por visitar a un profesional en salud mental, por otra parte es accesible para personas con limitaciones físicas, tímidas, con problemas de transportación, entre otros.

En la investigación de Flores (2012, pág. 22) cita a Yapero \& Speyer, (2010) quienes afirman que la relación terapéutica en línea es comparada con la presencial. Los profesionales de salud mental reportan que los clientes que usan consejería en línea son capaces de expresarse con mejor grado de información personal. El autor cita a Richards y Suler (2009) quienes describen un "efecto de desinhibición".

Este fenómeno es posible debido a que la gente se siente menos vulnerable al abrirse en un contexto anónimo sin enfrentar reacciones inmediatas. Siguiendo al mismo autor refiriéndose a la interacción (Yapher \& Speyer, 2010), se concluye que la consejería en línea provee dos métodos para la interacción con clientes, la comunicación asincrónica y la comunicación sincrónica.

En primer lugar, la comunicación asincrónica utiliza modalidades como: correo electrónico, mensajes y blogs, en donde el profesional y el cliente no se comunica de una forma (tándem) personal y fluida. Ningún participante tiene que estar en la computador al mismo tiempo, y cada uno puede considerar cuando y como contestar a la interacción.

Por otro lado, la comunicación sincrónica sucede cuando dos personas hablan o escriben de forma simultánea por medio de una conversación por chat, videoconferencia, etc. Los modelos de terapia actuales incluyen tecnología en línea aplicada en websites de información, test de diagnóstico psicoterapéutico, software, grupos de ayuda en línea, comunicación vía e-mail, uso de videoconferencias, chats, entre otros. Albornoz C, y 
otros (2007, pág. 25) destaca la "Agenda de Conectividad que tiene el gobierno ecuatoriano, para estimular el desarrollo social de los estratos sociales en condiciones de exclusión”.

El presente proyecto hará uso de este eje de trabajo para conectar a las personas que necesitan de una consejería virtual y no pueden visitar a un profesional en persona debido a que implica gastos, tiempo, etc.

Partiendo de la investigación de Brown (2012, págs. 4-56), en la que se analizan los problemas emocionales de los estudiantes en EEUU se observa los siguientes resultados en un estudio sobre consejería en línea y estudiantes de una universidad: El incremento de jóvenes con problemas de salud mental y la falta de recursos de servicios cara a cara. Aproximadamente del 12 al 18\% de estudiantes presentan diagnósticos de enfermedades mentales. Por lo que se evidencia la utilidad que aporta este sistema de consejería y el impacto que refleja en el mejoramiento de la salud mental de grupos humanos.

En nuestro país ha sido notable el crecimiento de usuarios de tecnología en general y de forma especial el uso de internet. Según el Ministerio de Ministerio de Telecomunicaciones en el 2013, 66 de cada100 personas usaron internet. Esta cifra es 11 veces mayor a la registrada en el 2006, donde tan solo eran 6 de cada 100 usuarios.

A pesar de este notable incremento de usuarios en la red, no se ha llevado a cabo proyectos que involucren a la tecnología en el área de consejería de Trabajo Social. En el ámbito nacional y local no se encuentran proyectos de investigación en esta área.

Los únicos proyectos que se acercan a la temática son: el proyecto de los Padres Salesianos en Azuay, donde para ayudar a mejorar la comunicación entre inmigrantes y familiares se instalaron cafés internet donde trabajan profesionales de la salud mental con el fin de mejorar la relación entre familiares; y el centro de escucha "Teléfono Amigo" en Quito, que ofrece un servicio telefónico donde las personas llaman para hablar sobre problemas que no pueden enfrentar, generalmente utilizan seudónimos. En este tipo de servicio ante todo son escuchados, pero no reciben consejería.

Tanto en la Universidad Técnica de Ambato como en las demás universidades del país no existe el servicio de consejería en línea, dado que no existe una metodología adecuada para su creación. Así mismo los profesionales tanto del área del Trabajo Social como del área de Psicología no cuentan con las orientaciones necesarias que permita desarrollar una Consejería en Línea de forma apropiada, desperdiciando de esta forma las ventajas que las nuevas tecnologías pueden aportar al proceso. 
Michalski (1995) define a las comunidades virtuales como "relaciones sociales unidas por un interés común circunstancias, crea productos y bienes colectivos, capital social en la red capital del conocimiento y ciencia”.

Tomando como referencia este concepto, el sistema de consejería en línea tendría como objeto crear una comunidad virtual entre los estudiantes y el DIBESAU, que brindaría soporte profesional basado en medios digitales y del mismo modo generaría una investigación científica sobre el mecanismo óptimo para llevar a cabo una Consejería en línea a través de procedimientos terapéuticos adecuados y la selección de herramientas informáticas que se ajusten a las necesidades de los profesionales del área de Psicología y Trabajo Social.

\section{Metodología.}

Se pretende organizar el proyecto en las siguientes etapas:

- Investigación de campo, que en términos de Graterol, (2012, pág. 26) "permite el conocimiento más a fondo del investigador, puede manejar los datos con más seguridad y podrá soportarse en diseños exploratorios, descriptivos $y$ experimentales, creando una situación de control en la cual manipula sobre una o más variables dependientes (efectos)", es decir que la investigación se da en su ambiente natural, por lo que se trabajará con los estudiantes a través del contacto directo.

- Levantamiento del diagnóstico del servicio de Consejería de la Universidad a través de la recolección de información, entrevistas con el personal a cargo y a estudiantes de varias carreras.

- Visita a las instituciones con proyectos afines o similares con la finalidad de recabar experiencias y opiniones de profesionales del área, que aporten sustancialmente al diseño de la metodología.

- Determinación de las variables que deberán ser probadas dentro de la investigación, así como los parámetros que la ejecución del proyecto. Las variables que inicialmente se han considerado son:

- Consejería en línea

- Problemas emocionales

Los métodos que se aplicarán sobre la posibilidad de abrir un sistema de consejería en línea son:

Inductivo. Mediante el análisis del diagnóstico del servicio de consejería actual y la percepción de un sistema de consejería en línea como línea base para la investigación.

Deductivo. De cómo la utilización de la consejería en línea en otros países puede también ser utilizada en el nuestro para reducir las brechas de exclusión en el 
acceso a servicios de salud mental. Se analizará las prácticas de consejería existentes actualmente tanto a nivel internacional como nacional, hasta llegar a la particularidad de nuestra realidad en la UTA

Como técnica para esta investigación, se aplicará el cuestionario de actitud acerca de servicios en línea, servicios cara a cara en el DIBESAU, dado a estudiantes de la modalidad presencial, se tomará en cuenta el sexo, la facultad, edad, que áreas necesitan más ayuda, que información pueden dar, que información educativa necesitan sobre las dos consejerías, si necesitan información sobre consejería en línea para nuevos estudiantes durante el periodo de orientación.

El procesamiento de la información obtenida en las diferentes fases de la investigación permitirá delinear la metodología de creación del Servicio de Consejería y el correcto uso de los instrumentos tecnológicos.

Finalmente se diseñará un modelo piloto en donde se realicen las pruebas necesarias tanto a nivel tecnológico como metodológico con la finalidad de validar la metodología diseñada.

\section{Conclusiones.}

- En los países latinoamericanos, no existe la consejería en línea en otros países de Norte América y Europa si ha estado presente para mejorar la práctica profesional, casi siempre en conjunción con la consejería en persona.

- Es de suma importancia la creación de una metodología sobre los servicios de salud mental en línea. Ayuda a incrementar y mejorar los servicios que el presente Departamento Estudiantil de la Universidad Técnica de Ambato ofrece a los estudiantes con problemas emocionales, esta metodología incorporará diagnósticos en las áreas de atención prioritaria de salud emocional de los estudiantes de la Universidad Técnica de Ambato, establecerá políticas, estrategias y procesos necesarios para realizar terapia en línea, aplicará el Método Científico de Investigación de Trabajo Social basado en evidencias de resultados de la intervención, y creará un proyecto piloto para validar la propuesta

\section{Referencias bibliográficas.}

Albornoz, C., Cabrera, V., \& Palacios, K. (2007). Los usos del Internet. Quito: Flacso.

Chloe, B. (2012). Online Counseling: Attitudes And Potential Utilization By College Students. Humboldt: Humboldt State University. 
Vol. 2 / No.3/ pág. 5-21. Julio-Septiembre/2018

WWW.visionariodigital.org

Flores, S. a. (2012). On line Counseling Mixed Methods of Investigation PDH Tesis. Texas: Universidad Corpus Cristy.

Graterol, R. (2012). Campus Virtual. Recuperado el 2014, de Mestría en Políticas Públicas: http://www.uovirtual.com.mx/moodle/lecturas/metoprot/10.pdf

Michalski, J. (1995). What is a virtual community? Spring N.12.

NASW (National Association of Social Workers). (Febrero de 2015). NASW.

Recuperado el 23 de Febrero de 2015, de http:nasw.

NASW. (Abril de 2007). Recuperado el Febrero de 2015, de http://www.naswdc-org

Raya Diez, E., \& Santolaya, M. (2009). The Information Society and its contribution to Social Work. Portularia, 10.

Tanrikulu, I. (2009). Counselors-in-training students' attitudes towards on line counseling. Elsevier, 4.

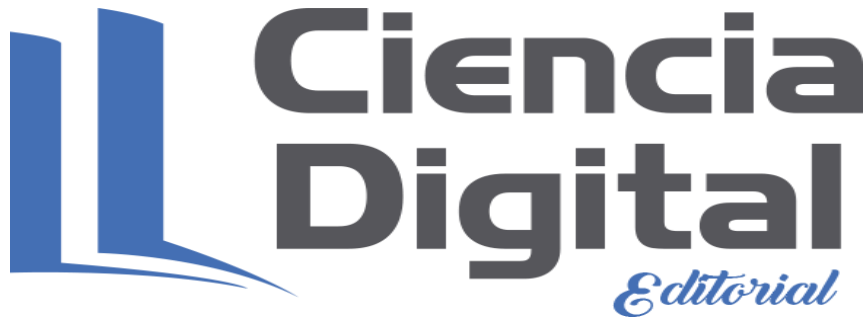




\section{Para citar el artículo indexado.}

Paredes T., Chiliquinga L., Quiroga M. \& Espín A. (2018). Sistema de consejería en línea para la Universidad Técnica de Ambato: una aproximación al tema. Revista electrónica Visionario Digital 1(1), 74-82. Recuperado desde:

http://cienciadigital.org/revistacienciadigital2/index.php/VisionarioDigital/article/view/ $\underline{91 / 85}$

\section{Ciencia}

El artículo que se publica es de exclusiva responsabilidad de los autores y no necesariamente reflejan el pensamiento de la Revista Ciencia Digital.

El articulo queda en propiedad de la revista y, por tanto, su publicación parcial y/o total en otro medio tiene que ser autorizado por el director de la Revista Ciencia Digital.
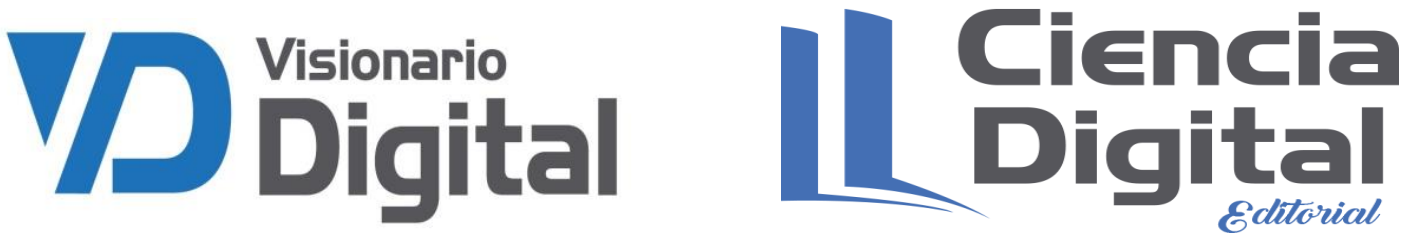